\title{
3D printed photonic structure for generation to zeroth- and high-order Bessel beams from a single-mode optical fiber
}

\author{
Innem V.A.K Reddy ${ }^{1,2}$, Andrea Bertoncini ${ }^{1}$, Carlo Liberale $^{1,3}$ \\ 1. Biological and Environmental Science and Engineering Division, King Abdullah University of Science and Technology, Saudi Arabia \\ 2. Department of Electrical Engineering, University at Buffalo, NY USA \\ 3. Computer, Electrical and Mathematical Science and Engineering Division, King Abdullah University of Science and Technology, Saudi
} Arabia

Bessel beams have gained prominence due to their diffraction-free propagation and self-healing properties [1]. The zeroth-order Bessel beam has maximum intensity at its center and has found many applications, including optical trapping and bio-imaging. On the other hand, higher-order Bessel beams have a node due to a phase singularity and carry orbital angular momentum (OAM). Over the past years, Bessel beams have been generated by using different approaches, namely by Fourier transforming a narrow circular beam with a lens, by using axicons and with holographic beam shaping techniques. The need to miniaturize optics has led to efforts towards the on-fiber generation of Bessel beams. The successful attempts include the fabrication of a customized annularcore fiber along with a polymer lens [2], or modifying the end of the fiber to an axicon profile with chemical or lithographic methods [3]. These techniques have limited capability on generating Bessel beams with on-demand optical parameters and are only focused on creating zeroth-order Bessel beams from optical fibers.

We present a new method for the generation of Bessel beams from optical fibers that utilizes stacked miniaturized optical elements 3D printed in a single step on the fiber facet with a commercial two-photon lithography based printer (Nanoscribe). The design allows for the generation of both zeroth- and high-order Bessel beams and a fully controllable tailoring of the beams' parameters, such as their propagation distance or the width of their central peak or node. Our structure includes a segment with a longitudinally varying annular-core photonic crystal fiber (PCF) design [4] used to transform the fundamental mode from the single-mode fiber into an annular beam, which is subsequently Fourier transformed with a microfabricated lens (Fig. 1). By designing the longitudinal variation of the annular-core PCF segment, the fundamental mode of the fiber is transformed into a beam ring of desired parameters. The structure includes also a PCF taper, used to modify the beam width output from the fiber to match the ring mode size in the annual-core PCF. For the generation of higher-order Bessel beams, a spiral phase plate (SPP) with the needed topological charge is also fabricated on the output face of the annular-core PCF. Finally, a microlens is fabricated to generate the desired Bessel beam at its focal plane. We demonstrate the generation high-quality Bessel beams with order starting from zero-order until 20 (Fig. 1).

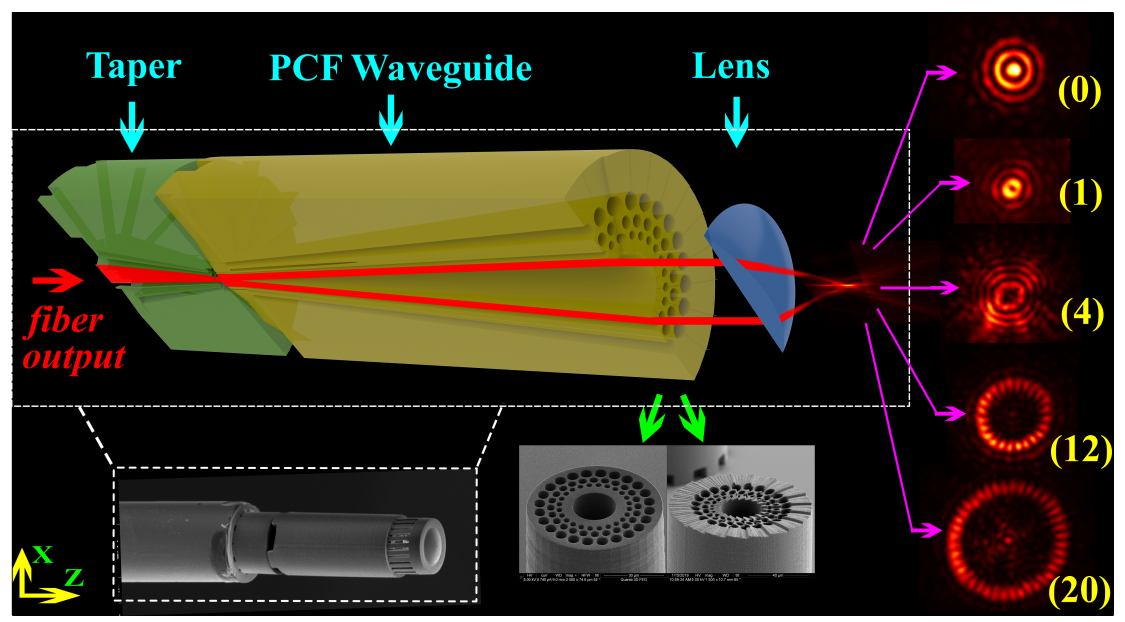

Figure 1: Rendered cross-section of the presented structure. In the insets, SEM images of the fabricated structure. On the right side, collected images of the generated 0-th and high-order Bessel beams.

\section{References}

[1] D. McGloin, K. Dholakia, "Bessel beams: diffraction in a new light," Contemporary Physics 46(1), 15-28, (2005).

[2] J. K. Kim, J. Kim, Y. Jung, W. Ha, Y. S. Jeong, S. Lee, A. Tunnermann and K. Oh, ”Compact all-fiber Bessel beam generator based on hollow optical fiber combined with a hybrid polymer fiber lens.” Optics Letters 34(19), 2973-2975, (2009).

[3] S. Cabrini, C. Liberale, D. Cojoc, A. Carpentiero, M. Prasciolu, S. Mora, V. Degiorgio, F. De Angelis and E. Di Fabrizio, "Axicon lens on optical fiber forming optical tweezers, made by focused ion beam milling," Microelectronic Engineering 83(4-9), 804-807, (2006).

[4] A. Bertoncini, C. Liberale, "3D printed waveguides based on photonic crystal fiber designs for complex fiber-end photonic devices," Optica 7, 1487-1494 (2020). 